\title{
Исследование влияния технологических факторов на неопределенность результатов измерения теплопроводности методом лазерной вспышки
}

\author{
(C) Е.С. Макарова, А.В. Асач, И.Л. Тхоржевский, В.Е. Фомин, А.В. Новотельнова, В.В. Митропов
}

Университет ИТМО,

197101 Санкт-Петербург, Россия

E-mail: makarova_helena_2011@mail.ru

Поступила в Редакцию 20 октября 2021 г.

В окончательной редакции 25 октября 2021 г.

Принята к публикации 25 октября 2021 г.

\begin{abstract}
Проведена оценка отклонения в измерениях теплопроводности методом лазерной вспышки для материалов с различным коэффициентом теплопроводности, возникающая вследствие наличия графитового покрытия на образце и малой толщины образца. Создана компьютерная модель метода в программной среде Comsol Multiphysics. Для массивных образцов при толщине графитового покрытия 20 мкм отклонение составляет 5.5\%. Толщина массивных образцов не влияет на результаты измерения. Для материалов с низкой теплопроводностью наблюдается резкий рост отклонения, достигающий $60 \%$. Для теплопроводящих материалов отклонение составляет $16-18 \%$. Для тонких образцов толщиной $<10$ мкм толщина графитового покрытия не влияет на результаты измерений. Определяющим является длительность лазерного импульса.
\end{abstract}

Ключевые слова: метод лазерной вспышки, теплопроводность, нормированное отклонение.

DOI: 10.21883/FTP.2022.02.51957.30a

\section{1. Введение}

Метод лазерной вспышки относится к нестационарным методам измерения теплопроводности. Он широко используется для измерения теплопроводности различных материалов, таких как керамика, металлы, полупроводники, термоэлектрики и другие функциональные материалы [1-4]. Для этого одну сторону образца облучают коротким лазерным импульсом, а затем на другой стороне регистрируют изменение температуры [5]. Используя полученные результаты измерений, зная значения плотности $(\rho)$ и удельной теплоемкости $\left(c_{P}\right)$ материала, можно определить коэффициент теплопроводности $(\lambda)$ образца по формуле

$$
\lambda=1.36976 \cdot \rho \cdot c_{p} \cdot \frac{h_{0}^{2}}{\pi^{2} \tau_{1 / 2}},
$$

где $h_{0}$ - высота образца, $\tau_{1 / 2}$ - время достижения половины максимальной температуры на обратной стороне образца.

Для данного метода существует ряд рекомендаций и ограничений, таких как конечность времени лазерного импульса [6], неравномерный нагрев образца, толщина, форма и непрозрачность образца $[7,8]$. В большинстве экспериментов для обеспечения наибольшего поглощения энергии импульса образцом и повышения точности измерения роста температуры образец покрывают слоем графита.

\section{2. Создание компьютерной модели}

Для оценки влияния различных факторов на отклонение измеренного коэффициента теплопроводности от истинного значения было создано несколько математических моделей, описывающих влияние на процесс измерения каждого из этих технологических факторов. Моделирование проводилось в программной среде Comsol Multiphysics.

Геометрическая модель исследуемого образца представлена в виде цилиндра с графитовым покрытием (рис. 1). В компьютерной модели рассматривался однородный изотропный образец с фиксированным значением коэффициента теплопроводности, диаметром $d=30$ мм и высотой $h_{0}=2$ мм. Графитовый слой толщиной $h_{1}$ покрывал всю поверхность образца. Нижняя сторона образца облучалась импульсом лучистой энергии, имеющим распределение по времени в форме Гаусса. Измерения моделировались в атмосфере обдувки газообразным азотом.

Поток энергии от лазерного импульса распространяется в объеме образца по закону Фурье:

$$
\mathbf{q}=-\lambda \nabla T
$$

что приводит к повышению температуры на противоположной стороне образца. Здесь $\mathbf{q}-$ вектор плотности теплового потока, $T$ - температура.

Распределение температуры в заданной области пространства и ее изменение во времени описывается основным уравнением теплопроводности:

$$
\rho c_{p} \frac{\partial T}{\partial t}+\nabla \cdot \mathbf{q}=0 .
$$

Граничные условия для облучаемой поверхности:

$$
-\mathbf{n} \cdot \mathbf{q}=q_{b},
$$


где $\mathbf{n}$ - вектор нормали к поверхности теплообмена, $q_{b}$ - плотность теплового потока от импульса лучистой энергии. Для остальных поверхностей было использовано граничное условие радиационного теплообмена и конвекционного теплообмена:

$$
\begin{gathered}
-\mathbf{n} \cdot \mathbf{q}=\varepsilon \sigma\left(T_{\mathrm{amb}}^{4}-T^{4}\right), \\
q_{0}=\alpha \cdot\left(T_{\mathrm{ext}}-T\right) .
\end{gathered}
$$

Здесь $\sigma$ - постоянная Стефана-Больцмана, $\varepsilon-$ степень черноты тела, $T_{\mathrm{amb}}$ - температура окружающей среды, $T_{\text {ext }}$ - температура газообразного азота. Значение $\varepsilon$ принималось равным 0.9 для всех поверхностей образцов. Значение $T_{\mathrm{amb}}$ было принято равным $293.15 \mathrm{~K}$.

Для горизонтальных пластин, обращенных теплопередающей поверхностью вверх, коэффициент теплоотдачи $\alpha$ можно рассчитать как [9]:

$$
\begin{gathered}
\alpha=1.3 \cdot \frac{N u \cdot \lambda_{f}}{l_{0}}, \\
N u=C \cdot R a^{m},
\end{gathered}
$$

где $R a$ - число Релея, которое определяется теплофизическими свойствами газообразного азота, перепадом температур и определяющим размером и для данной модели $R a=5469$. $C$ и $m-$ коэффициенты, определяемые в зависимости от режима движения окружающей среды (для нашего случая $C=0.54$ и $m=0.25$ ), $\lambda_{f}-$ коэффициент теплопроводности газообразного азота при средней температуре среды, $l_{0}$ - определяющий размер (для диска определяющим размером является его диаметр $d$ ).

На основании исходных данных расчетное значение коэффициента теплоотдачи составило $\alpha=5.1 \mathrm{BT} /\left(\mathrm{M}^{2} \cdot \mathrm{K}\right)$.

В модели задавалась длительность лазерного импульса $t_{\text {imp }}=2$ мс, что соответствует заводским параметрам установки NETZSCH LFA 457 MicroFlash. Мощность импульса подбиралась такой, чтобы изменение температуры на обратной стороне образца не превышало 2 K.

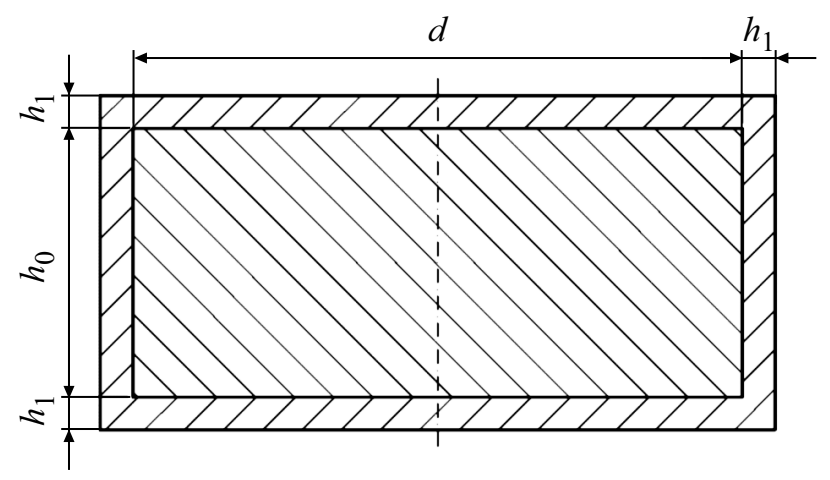

Рис. 1. Геометрическая форма образца в модели: $h_{0}$ - высота образца, $h_{1}$ - толщина графитового покрытия, $d-$ диаметр образца.

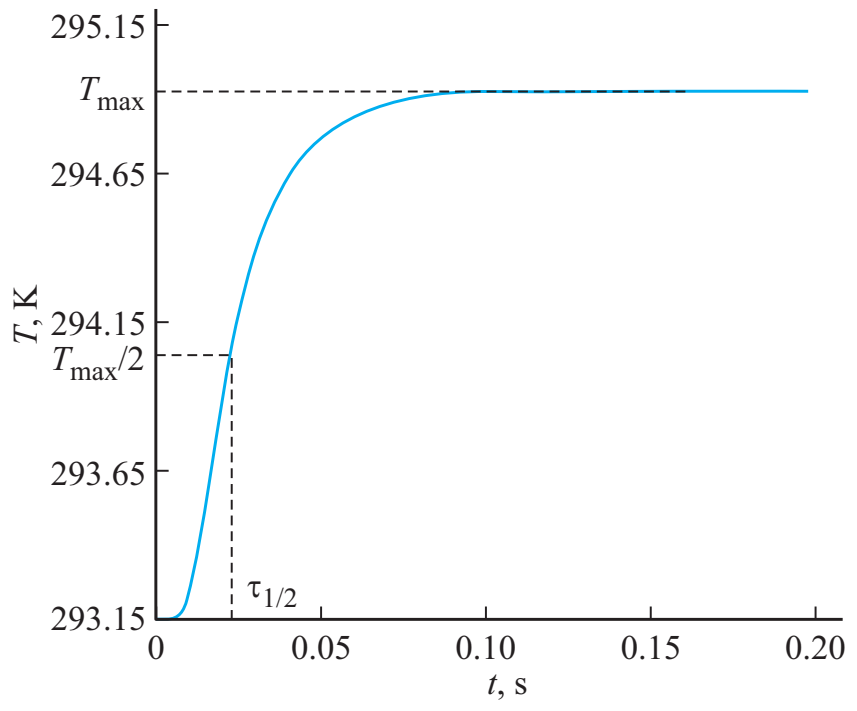

Рис. 2. Временна́я зависимость температуры обратной стороны образца, полученная с помощью компьютерного моделирования.

На основе математического моделирования были получены зависимости роста температуры на обратной стороне образца от времени (рис. 2). Ожидаемое понижение температуры за счет теплоотдачи в окружающую среду наступает существенно позже моделируемого промежутка времени и, соответственно, на графике не отображается. Эти данные в дальнейшем были использованы для определения значения $\left(\tau_{1 / 2}\right)$, которое необходимо для расчета коэффициента теплопроводности $\left(\lambda_{c}\right)$ по формуле (1). Полученное расчетное значение коэффициента теплопроводности сравнивалось с истинным коэффициентом $\left(\lambda_{t}\right)$, задаваемым на стадии моделирования. Таким образом проводилась оценка влияния рассматриваемых факторов на точность измерения.

Для наглядного отображения отличий истинного и расчетного значений коэффициентов теплопроводности определялась их разность и проводилась нормировка по истинному значению:

$$
\delta=\frac{\lambda_{t}-\lambda_{c}}{\lambda_{t}} \cdot 100 \%
$$

\section{3. Результаты и обсуждение}

\section{1. Массивные образцы}

В работе [10] было проведено исследование и выявлено, что толщина графитового покрытия колеблется в пределах от 5 до 15 мкм с одной стороны нанесения на образец. При исследовании массивных образцов влиянием этого слоя на результат измерительного процесса обычно пренебрегают. Однако графитовый слой может внести вклад в оценку измерений. Поэтому нами было рассмотрено влияние толщины графитового покрытия, а 

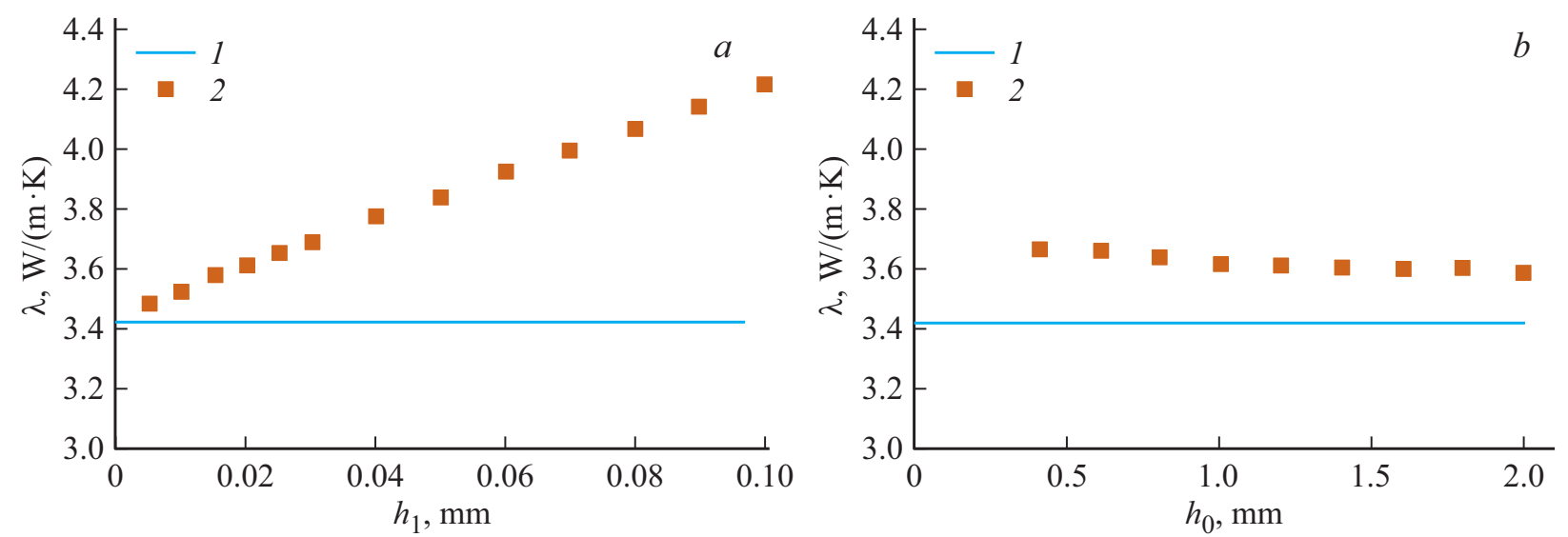

Рис. 3. Зависимость коэффициента теплопроводности от толщины графитового покрытия $h_{1}(a)$; высоты образца $h_{0}(b)$. 1 - истинное значение $\lambda_{t}, 2$ - расчетное значение $\lambda_{c}$.

также влияние толщины самого образца на результаты измерения теплопроводности образцов. Рассматриваемые образцы имели фиксированное значение коэффициента теплопроводности $\lambda=3.4 \mathrm{BT} /(\mathrm{M} \cdot \mathrm{K})$.

3.1.1. Влияние толщины графитового покрытия. Высота образцов первой серии составляла $h_{0}=2$ мм, и их поверхность покрывалась графитовым слоем различной толщины $h_{1}$. Результаты моделирования представлены на рис. 3, $a$. На основе этих результатов было установлено, что с увеличением толщины графитового покрытия наблюдается линейный рост расчетного значения теплопроводности. При толщине слоя графита 100 мкм отклонение $\delta$ расчетного значения от истинного достигает 20\%. Для толщины графитового покрытия 20 мкм, что близко к реальной толщине графитового покрытия в натурных экспериментах, отклонение составляет $5.5 \%$. В дальнейших исследованиях была использована средняя толщина графитового покрытия $h_{1}=20$ мкм.

Такое поведение расчетного значения коэффициента теплопроводности связано с высоким (более чем в 30 раз) коэффициентом теплопроводности графита.

3.1.2. Влияние толщины образца. Во второй серии численных экспериментов варьировалась высота образца $h_{0}$ в диапазоне от 0.4 до 2.0 мм. Толщина слоя графитового покрытия принималась постоянной и равной 20 мкм. Результаты моделирования представлены на рис. $3, b$. На основании полученных данных можно сделать вывод о том, что толщина образца практически не влияет на результаты измерений. Рост отклонения наблюдается лишь при толщинах образца ниже $0.7 \mathrm{MM}$, что является нижним значением рекомендованной толщины образца для установки NETZSCH LFA 457 MicroFlash.

Из формулы (1) видно, что расчетное значение теплопроводности прямо пропорционально квадрату толщины образца. С увеличением графитового покрытия увеличивается общая толщина образца, которая не учитывается в расчетной формуле, что приводит к росту неопределенности на рис. $3, a$.

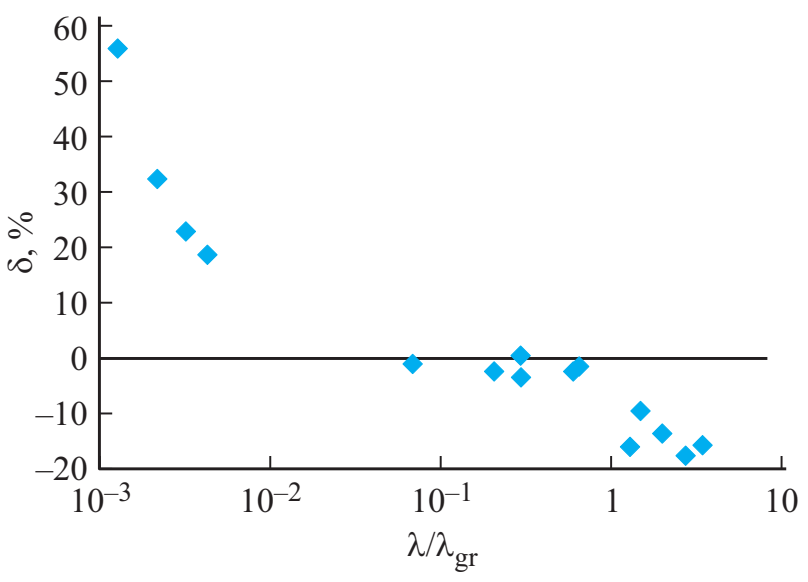

Рис. 4. Зависимость нормированного отклонения измерений коэффициента теплопроводности $\lambda$ массивных образцов от свойств материала.

3.1.3. Влияние материала образца. Оценка влияния графитового покрытия на результаты измерений проводилась для материалов с различными значениями коэффициента теплопроводности. Были рассмотрены материалы с коэффициентом теплопроводности от $0.15 \mathrm{BT} /($ м $\cdot \mathrm{K})$ (полиимид) до $400 \mathrm{BT} /($ м $\cdot \mathrm{K})$ (медь). Свойства использованных в ходе моделирования веществ представлены в таблице. Образцы имели одинаковые высоту $h_{0}=2$ мм и толщину слоя графитового покрытия $h_{1}=20$ мкм.

Результаты моделирования представлены на рис. 4. Проведенное моделирование показало, что для материалов с коэффициентом теплопроводности от $0.01 \lambda_{\mathrm{gr}}$ и ниже наблюдается резкий рост $\delta$, который достигает $60 \%$ для полиимида с теплопроводностью $0.15 \mathrm{BT} /(\mathrm{M} \cdot \mathrm{K})$. Такую зависимость можно объяснить наличием тонкого слоя графита на боковой поверхности образца, который в данном случае играет роль теплового моста. На участке от 0.1 до 1 отклонение $\delta$ минимально и сопоста- 
Свойства веществ, используемых в компьютерной модели

\begin{tabular}{l|c|r|c}
\hline & $c_{P}$, Дж/(кг $\left.\cdot \mathrm{K}\right)$ & $\rho$, кг $/ \mathrm{M}^{3}$ & $\lambda, \mathrm{BT} /(\mathrm{M} \cdot \mathrm{K})$ \\
\hline Полиимид & 1100 & 1300 & 0.15 \\
Нейлон & 1700 & 1150 & 0.26 \\
Полиэтилен & 1900 & 930 & 0.38 \\
Слюда & 880 & 2900 & 0.5 \\
Висмут & 122.08 & 9800 & 7.8 \\
Сурьма & 207 & 6684 & 24.3 \\
Оксид алюминия & 730 & 3965 & 35 \\
Свинец & 127 & 11340 & 35.3 \\
Платина & 133 & 21450 & 71.6 \\
Железо & 440 & 7870 & 76.2 \\
Вольфрам & 132 & 19350 & 174 \\
Алюминий & 894 & 2700 & 236 \\
Золото & 129 & 19300 & 317 \\
Медь & 385 & 8940 & 400
\end{tabular}

вимо с заявленной точностью измерительной установки. Для материалов, теплопроводность которых превышает теплопроводность графита, наблюдается увеличение $\delta$ с изменением его знака, так как слой графита для таких случаев ведет себя как теплоизолятор.

\section{2. Тонкие образцы}

Отдельно были рассмотрены тонкие образцы, которые не относятся к тонкопленочным структурам, но их толщина ниже рекомендованной производителем измерительного оборудования (10-100 мкм). При толщине образца в несколько десятков микрометров наличие графитового покрытия может оказать существенное влияние на результаты эксперимента. Для их оценки было проведено численное моделирование метода лазерной вспышки для образцов толщиной от 10 до 70 мкм. Рассматриваемые образцы имели фиксированное значение коэффи-

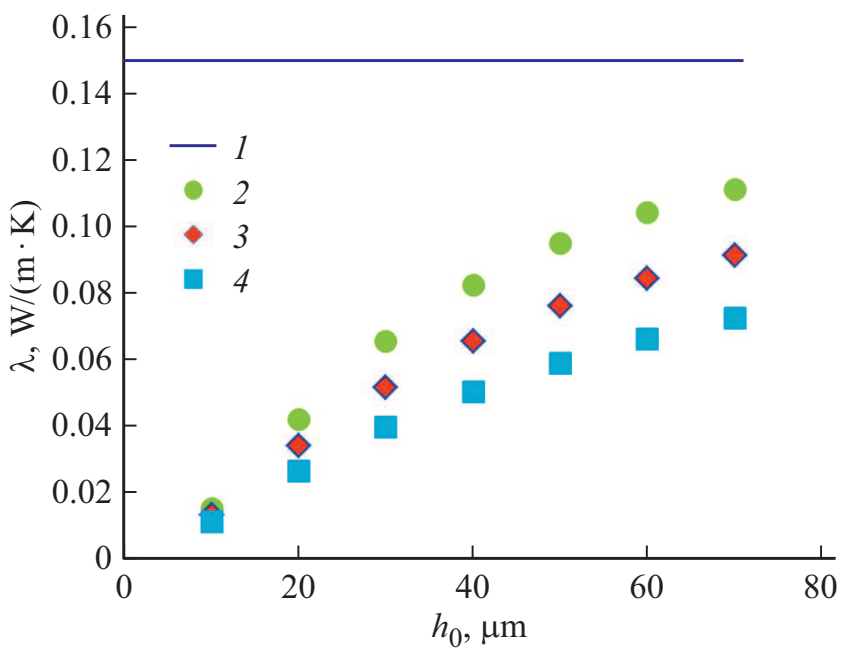

Рис. 5. Зависимость коэффициента теплопроводности тонких пленок от толщины образца $h_{0} .1-$ истинное значение $\lambda_{t}$. Толщина графитового покрытия $h_{1}$, мкм: $2-4,3-10,4-20$. циента теплопроводности $\lambda=0.15 \mathrm{BT} /(\mathrm{M} \cdot \mathrm{K})$. Толщина графитового покрытия изменялась от 4 до 20 мкм. Результаты этого моделирования представлены на рис. 5 . Проведенное моделирование показало, что наибольшее отклонение вычисленного коэффициента теплопроводности от истинного наблюдалось при наименьшей толщине образца (10 мкм). При этом толщина графитового покрытия $h_{1}$ не влияет на полученные значения. Для такой толщины становится более существенным влияние конечности импульса, чем толщины графитового покрытия, так как время измерения температурного отклика на обратной стороне образца становится соизмеримым с длительностью лазерного импульса $[6,11]$.

Для увеличения времени нагрева образца можно увеличить эффективную толщину образца за счет смещения детектора на некоторое расстояние от центра нагрева, как это предложено в работах $[12,13]$. Графитовое покрытие начинает играть существенную роль для образцов с толщиной 20 мкм и более.

Также из графика видно, что наиболее точные измерения теплопроводности получились при наименьшем слое графита, 4 мкм, и наибольшей толщине образца, что согласуется с предыдущими результатами. Для экспериментального исследования необходимо искать методы более тонкого нанесения графитового покрытия.

\section{4. Заключение}

Анализ результатов моделирования показал, что графитовое покрытие толщиной до 20 мкм незначительно влияет на результаты эксперимента для массивного образца. Однако повторное покрытие образца графитовым слоем с увеличением его толщины до 100 мкм приводит к увеличению отклонения в измерениях до $20 \%$.

Толщина массивных образцов не влияет на результаты измерений. Отклонение становится заметным с уменьшением толщины $<0.7$ мм. Для массивных образцов с малым значением коэффициента теплопроводности $(<1 \mathrm{BT} /(\mathrm{M} \cdot \mathrm{K}))$ отклонение измеренного значения коэффициента теплопроводности $\lambda$ методом лазерной вспышки может достигать 60\%. Это можно объяснить наличием тонкого слоя графита на боковой поверхности образца, который играет роль теплового моста, что приводит к завышению значения коэффициента теплопроводности. Для материалов с коэффициентом теплопроводности выше, чем у графита, тоже наблюдается увеличение нормированного отклонения, только с другим знаком, свидетельствующим о том, что наличие графитового покрытия приводит к занижению измеряемого коэффициента теплопроводности.

Для тонких образцов вклад графитового покрытия может оказаться существенно выше (до 90\%). Также для тонких образцов существенным параметром, влияющим на результаты измерений, будет его толщина. При толщине образца ниже 10 мкм толщина графитового покрытия не будет оказывать существенного влияния на 
результаты измерения. Определяющим фактором будут мощность и длительность лазерного импульса лучистой энергии, подаваемого на образец.

Проблемы, возникающие при измерении тонких образцов методом лазерной вспышки, связаны с искажением кривой нагрева лазерным импульсом вследствие наложения температурной кривой нагрева и самого импульса. Для увеличения времени нагрева образца можно увеличить эффективную толщину образца за счет смещения детектора на некоторое расстояние от центра нагрева.

При измерении тонких образцов необходимо, по возможности, отказаться от дополнительных покрытий или контролировать толщину графитового покрытия для ее дальнейшего учета при измерениях.

\section{Финансирование работы}

Исследование выполнено при финансовой поддержке РФФИ в рамках научного проекта № 20-32-90210.

\section{Конфликт интересов}

Авторы заявляют, что у них нет конфликта интересов.

\section{Список литературы}

[1] K. Shinzato, T. Baba. J. Thermal Analysis and Calorimetry, 64 (1), 413 (2001).

[2] T. Baba, N. Taketoshi, T. Yagi. Jpn. J. Appl. Phys., 50 (11), 11RA01 (2011).

[3] A. Cezairliyan, T. Baba, R. Taylor. Int. J. Thermophys., 15, 317 (1994).

[4] M. Akoshima, T. Baba. Int. J. Thermophys., 27, 1189 (2006).

[5] W.J. Parker, R.J. Jenkins, C.P. Butler, G.L. Abbott. J. Appl. Phys., 32, 1679 (1961).

[6] S.F. Corbin, D.M. Turriff. In book: Characterization of Materials, 510 (2012).

[7] L. Vozar, W. Hohenauer. Int. J. Thermophys., 26 (6), 1899 (2005).

[8] А.В. Асач, Г.Н. Исаченко, А.В. Новотельнова, В.Е. Фомин, К.Л. Самусевич, И.Л. Тхоржевский. ФТП, $53(6), 731$ (2019).

[9] М.А. Михеев, И.М. Михеева. Основы теплопередачи (М., Энергия, 1977).

[10] K.H. Lim, S.K. Kim, M.K. Chung. Thermochimica Acta, $494(1-2), 71$ (2009)

[11] K.B. Larson, K. Koyama. J. Appl. Phys., 39 (9), 4408 (1968).

[12] H. Ohta, H. Shibata, Y. Waseda. Rev. Scientific Instrum., 60 (3), 317 (1989).

[13] H. Shibata, H. Ohta, Y. Waseda. Mater. Trans., JIM, 32|,(9), 837 (1991).

Редактор Г.А. Оганесян

\section{Investigation of the influence of technological factors on the uncertainty of the results of measuring thermal conductivity by the method of laser flash}

\author{
E.S. Makarova, A.V. Asach, I.L. Tkhorzhevsky, \\ V.E. Fomin, A.V. Novotelnova, V.V. Mitropov \\ ITMO University, \\ 197101 St. Petersburg, Russia
}

Abstract The estimation of the deviation in the measurements of thermal conductivity by the laser flash method for materials with different thermal conductivity coefficients, arising due to the presence of a graphite coating on the sample and the small thickness of the sample, is carried out. A computer model of the method was created in the Comsol Multiphysics software environment. For bulk samples with a graphite coating thickness of $20 \mu \mathrm{m}$, the deviation is $5.5 \%$. The thickness of bulk samples does not affect the measurement results. For materials with low thermal conductivity, a sharp increase in the deviation is observed, reaching $60 \%$. For thermally conductive materials, the deviation is $16-18 \%$. For thin samples less than $10 \mu \mathrm{m}$ thick, the thickness of the graphite coating does not affect the measurement results. The decisive factor is the duration of the laser pulse. 